\title{
Synchronization of Lorenz System Based on Fast Stabilization Sliding Mode Control
}

\author{
Xu Guowei, ${ }^{1}$ Wan Zhenkai, ${ }^{2}$ and Li Chunqing ${ }^{1}$ \\ ${ }^{1}$ School of Computer Science and Software Engineering, Tianjin Polytechnic University, Tianjin, China \\ ${ }^{2}$ Information Technology Center, Tianjin Polytechnic University, Tianjin, China \\ Correspondence should be addressed to Xu Guowei; guoweixu0529@sina.com
}

Received 14 August 2014; Accepted 13 October 2014

Academic Editor: Xudong Zhao

Copyright (c) $2015 \mathrm{Xu}$ Guowei et al. This is an open access article distributed under the Creative Commons Attribution License, which permits unrestricted use, distribution, and reproduction in any medium, provided the original work is properly cited.

\begin{abstract}
A sliding mode control approach is achieved for Lorenz system based on optimal finite time convergent and integral sliding mode surface. The system perturbation is divided into two parts: the unmatched and the matched parts. Firstly, we design a discontinuous control for the unmatched part which will not be amplified. Secondly, we design a continuous control, that is, the ideal control to stabilize the Lorenz system error states in finite time stabilization. Then the controller based on integral sliding mode is constructed to ensure the robustness. The proposed method is proven to guarantee the stability and the robustness using the Lyapunov theory in the system uncertainties and external perturbation. Finally, the numerical simulations demonstrate that the proposed controller is effective and robust with respect to the perturbation.
\end{abstract}

\section{Introduction}

In the past twenty years, chaotic synchronization has attracted more and more attention among scientists of different fields including secure communication, chemical reactions, biology, neural network, mechanics, and ecological systems [1-3]. Reference [4] has proposed an exponential synchronization method with mixed time delays for a class of uncertain master and slave neural networks, where the delays include distributed time delays and discrete and different neutral. The norm-bounded uncertainties and the polytypic uncertainties are separately considered. Some delaydependent sufficient conditions are given in the sense of freeweighting matrices and discretized Lyapunov-Krasovskii functional. Due to the significance of attenuating chaotic synchronization with uncertainties in practical applications, combination of techniques with time-delays theory has been proposed in [5-9]. Since the modern systems become more and more complicated, physical model is usually hard to obtain in practice. Related control should be more considered under the data-driven framework. Many papers are available about recent model-based and data-driven control scheme [10-12].
Sliding mode control is developed as a very efficient tool for its fine robustness to control the system plants operating against disturbances and parameter uncertainties. It is robust with respect to external disturbances and model uncertainties satisfying the matching condition, roughly speaking, to guarantee effective elimination of uncertainties of system or external disturbances; obviously they should play a part in the same subspace as an admissible control [9-13]. The design of sliding mode control approach is usually composed of the following two steps [14]. First, we design a sliding mode surface such that the state trajectory in sliding mode satisfies the design specifications. Second, a control function is designed making the switching surface attractive to the desired system equilibrium state in the presence of external disturbances or uncertainties.

However, traditional sliding mode control method has two main disadvantages.

(1) The traditional sliding mode controllers cannot attenuate unmatched disturbances.

(2) Only asymptotical convergence is ensured for the designed traditional sliding mode controller rather than the finite time convergence. 
An effectively robust technique is sliding mode control (SMC) system, because of its ability to withstand external disturbances and model uncertainties which can satisfy the matching condition. The design solution trajectory is not robust for the unmatched disturbances. Note that this condition fails a sliding mode enforcing can only partially reject the disturbances of system for chaotic synchronization. The purpose of the design of a sliding mode controller effectively minimizes the unmatched disturbance in a robust way.

Unmatched disturbances widely exist in various control systems; otherwise, system states from the sliding surface to the desired set point are asymptotical stabilization rather than finite time stabilization. In order to solve this problem, finite time control is studied to drive the controlled systems to satisfy their design specifications in finite time of optimal control and $[15,16]$ propose an integral sliding mode control (ISMC) surface concept. The purpose to extend the ISMC method can handle the unmatched condition with external disturbances or model uncertainties.

In this paper, we design a novel ISMC to guarantee the sliding mode motion in finite time convergence under the presence of unmatched disturbances through combining optimal control and integral sliding surface. The novel sliding mode controller based on ISMC surface method has better performance in finite time convergence, robustness, and stationary. Otherwise, the disturbances do not need to satisfy the matched condition. The design of SMC law based on finite time stabilization is easy to be realized in the presence of unmatched disturbance.

The outline of this paper is organized as follows. In Section 2, the Lorenz system and assumptions are introduced. In Section 3, the integral sliding manifold and perturbation decomposition is discussed. The design of sliding mode control based on finite time stabilization is developed and ISMC is resolved in Section 4. Finally, the proposed sliding mode control method is presented to verify the effectiveness in Section 5 and some summarized conclusions are made in Section 6.

\section{Problem Formulation}

The Lorenz system is first proposed to descript the unpredictable behavior of the weather in 1963. Recently, several different physical systems such as laser devices, disk dynamics, and several problems can be described by Lorenz equations which related to convection. Consider the following Lorenz system is depicted by:

$$
\dot{x}=A x+f(x)
$$

where $x \in R_{n}$, is the state and

$$
A=\left(\begin{array}{ccc}
-10 & 10 & 0 \\
28 & -1 & 0 \\
0 & 0 & -\frac{8}{3}
\end{array}\right), \quad f(x)=\left(\begin{array}{c}
0 \\
-x_{1} x_{3} \\
x_{1} x_{2}
\end{array}\right)
$$

where $x \in R_{n}$, are state variables and which has a chaotic attractor as shown in the course of time (Figure 1).
A control input $u$ is proposed to be added in order to effectively control the system (1). Considering external disturbances or system uncertainties and adding this control input, the controlled system equation can be described by

$$
\dot{y}=A y+f(y)+\Delta f+B u+\delta(t)
$$

where $u \in R$ is the control input and $\Delta f$ model uncertainties and $\delta(t)$ is an external disturbance. The synchronization problem wants to design an ISMC $u$ based on finite time stabilization, which synchronize the states of the master system (1) and the slave system (3) in spite of the unknown nonlinear uncertainties or external disturbances. In other words, the aim of synchronization is to make

$$
\lim _{t \rightarrow \infty}\|y-x\|=0
$$

Define the synchronization error of the master system and the slave system as $e=y-x$. The tracking error system is defined as

$$
\dot{e}=A e+f(y)-f(x)+B u+\phi,
$$

where $\phi$ is a perturbation, and $\phi=\Delta f(y)-\Delta f(x)+\delta(t)$.

Therefore, we design a sliding mode controller based on finite time stabilization for given chaotic uncertain Lorenz system (3) so that the finite time stability of the resulting error system (5) can be obtained in the sense of (4).

The following assumption is derived as the following main results.

Assumption 1. The actual value of $\phi$ is bounded with a known function $\Psi(x, t) \in L_{\infty}$; that is, $\phi(x, t) \leq \Psi(x, t)$ for all $x$ and $t$.

Assumption 2. The matrix product $G B$ is invertible.

Assumption 3. Consider $|f(y)-f(x)|<\alpha<\infty$.

Generally speaking, because the trajectory of chaotic systems is always bounded, it is reasonable and not restrictive in Assumption 3.

This paper proposes a new sliding mode controller for the master-slave Lorenz chaotic systems with the presence of unmatched disturbance to guarantee stable synchronization in finite time.

\section{Integral Sliding Manifolds for Lorenz System}

In consequence, a design of sliding mode control based on optimal control and ISMC surface in finite time convergence is designed for chaotic synchronization under the presence of unmatched disturbance. There exist two major phases; firstly, an appropriate sliding manifold is selected, such that the sliding motion on the surface has the desired properties. Secondly, the robustness of sliding mode controller based on integral sliding mode can be guaranteed in finite time stabilization. 

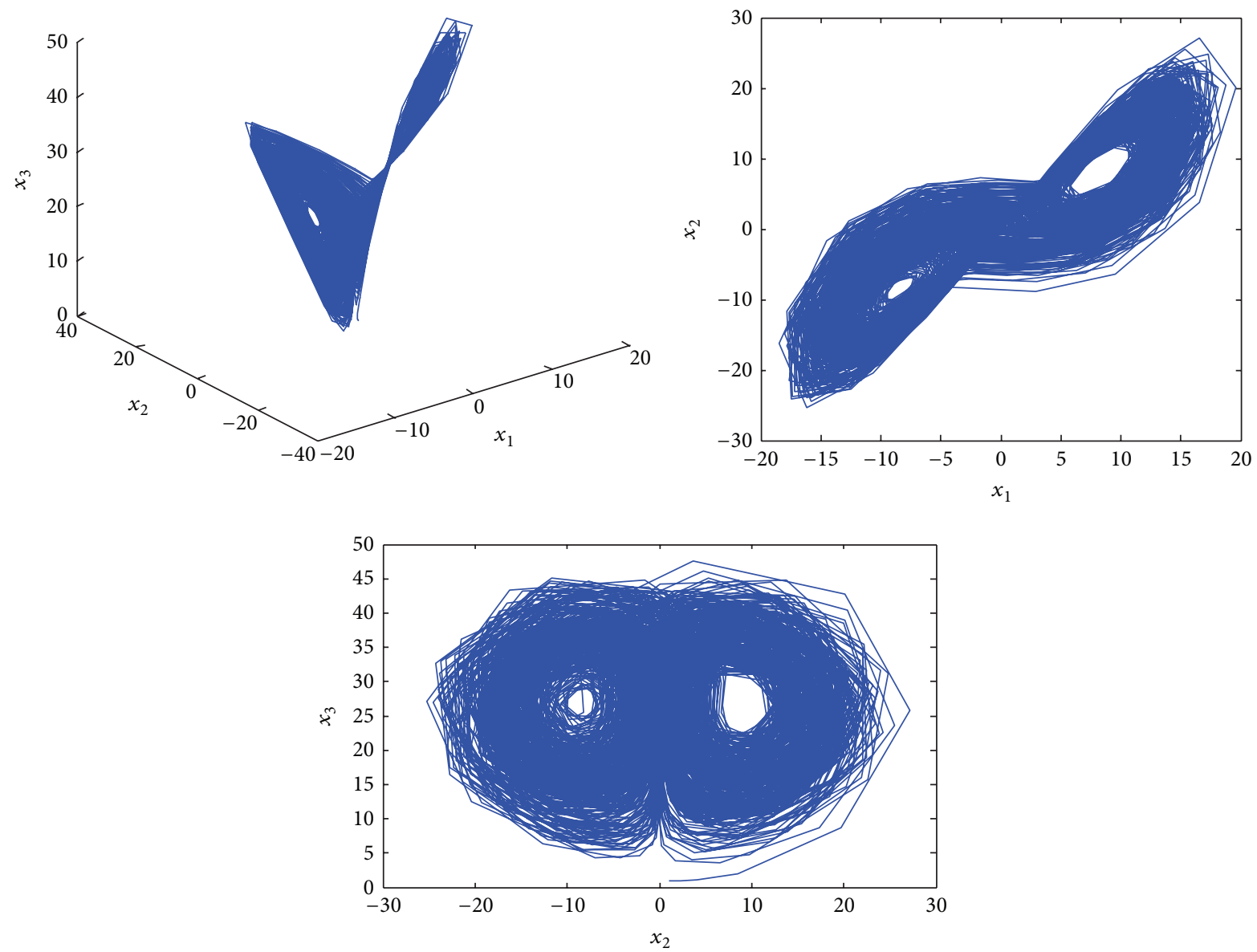

FIGURE 1: The Lorenz system chaotic response for system (1).

The sliding mode surface is described as

$$
s(t)=G e(t)-\int_{t_{0}}^{t}\left[G A e(\tau)+u_{0}\right] d \tau,
$$

where $u_{0}$ is a part of control input to be determined later so that the states can fit the performance of control in the sliding mode.

We expect the ISMC surface (6) to converge to zero in finite time. However, when sliding mode surface is converged to zero in finite time, we must study the movements of closedloop system in the ISMC surface (6). Thereby we should analyze the behaviors of error system (5) under the ISMC surface in this section.

The following identity $I_{n}=B B^{+}+B^{\perp} B^{\perp+}$ always holds for any matrix $B \in R^{n \times n}$, where the left inverse of $B$ is defined as $B^{+}=\left(B^{T} B\right)^{-1} B^{T}$, and we can use the columns of $B^{\perp} \epsilon$ $R^{n \times(n-m)}$ to span the null space of $B^{T}$. From the above analysis, the perturbation of Lorenz system (1) can be rewritten as

$$
\phi=B B^{+} \phi+B^{\perp} B^{\perp+} \phi
$$

and $B^{+} B^{\perp}=0$. Taking the derivative of (6) about time $t$ along with the synchronous error system (5), one can obtain the following:

$$
\dot{s}=G(f(y)-f(x))+B u+G \phi-K e .
$$

Order $\dot{s}=0$; the equivalent control from (7) is gotten as follows:

$$
u_{\mathrm{eq}}=K e-G(f(y)-f(x))-G \phi .
$$

One can obtain from substituting $u_{\mathrm{eq}}$ into the original system (5)

$$
\begin{aligned}
\dot{e}= & A e+(f(y)-f(x))+B u_{0} \\
& +\left[\left(I-B(G B)^{-1} G\right)\right] B^{\perp} B^{\perp+} \phi .
\end{aligned}
$$

Remarks 4-6 can be inferred.

Remark 4. In [16], setting $G=B^{+}$can be guaranteed which makes that the residual perturbation norm $[(I-$ $\left.\left.B(G B)^{-1} G\right)\right] B^{\perp} B^{\perp+} \phi$ is the most minimal. Moreover, using $B^{+} B^{\perp}=0$, the term of residual perturbation changes $B^{\perp} B^{\perp+} \phi$; that is, the unmatched perturbation $B^{\perp} B^{\perp+} \phi$ is left unchanged. So we cannot compensate the unmatched perturbation for error system (5) which would only result in amplification for any attempt to compensate.

Remark 5. We can obtain that the squared norm of the term of $B^{\perp} B^{\perp+} \phi$ is smaller than the original perturbation which we can obtain from orthogonality $\left\|B^{\perp} B^{\perp+} \phi\right\|^{2}=\|\phi\|^{2}-\left\|B B^{+} \phi\right\|^{2}$. 
Remark 6. The existence of equivalent controller is proved which does not require to specifically obtain equivalent controller. Moreover, $\phi(x, t)$ is unknown, but it will not affect the existence of the equivalent control.

\section{Design of ISMC Controller Based on Finite Time Stabilization}

In this section, we design a sliding mode controller $u$ to ensure that the states of closed-loop error system step into the ISMC surface (6) from arbitrary initial values. The ISMC control law based on finite time stabilization is divided into two parts. The first one is named ideal control which is continuous part and stabilizes the normal system (5) in finite time stabilization without considering any perturbation. The second part is named ISMC which gives the perturbation compensation for system (5) and ensures that the system states obtain stabilization in finite time.

The sliding mode control law for Lorenz system is defined as follows:

$$
u=u_{0}+u_{1}
$$

where the ideal control is $u_{0}$ and stabilizes the system states in finite time without any perturbation for system (5). In order to ensure the sliding mode motion on the sliding surface, the control law $u_{1}$ is designed for $t>0$ with system uncertainties.

The purpose of control design is to drive the Lorenz system error states of (5) to $e(t)=0$ at a fixed final time $t=t_{F}<\infty$, where $e(0)$ is an initial state condition and is bounded. We design a sliding mode control law using ISMC surface and optimal control laws. Moreover, the designed sliding mode control law is finite time stabilization based on the optimal closed-loop controller.

The following minimization criterion is used to ensure finite time stabilization:

$$
J=\frac{1}{2} \int_{0}^{t_{F}}\left(e^{T} Q e+u_{0}^{2}\right) d t
$$

with $t_{F}<\infty$, and $Q$ is a symmetric positive definite matrix which is constrained in the fixed final error state $e\left(t_{F}\right)=0$.

Theorem 7 (see [17]). The following linear system is considered:

$$
\dot{e}=A e+B u,
$$

where $(A, B)$ is reachable. The continuous control law $u_{0}$ is used to minimize criteria (12) and drive system (13) to $e(t)=0$ at point $t=t_{F}$ which satisfies initial bounded condition e(0) (with $\left.0<t \leq t_{F}<\infty\right)$ as

$$
u_{0}=-B^{T} P e(t)+B^{T} \delta(t),
$$

where $\delta(t)$ and $P$ are defined as

$$
\begin{gathered}
\dot{\delta}=-\left(A^{T}-P B B^{T}\right) \delta \\
P A+A^{T} P-P B B^{T} P+Q=0 .
\end{gathered}
$$

The control law $u_{0}$ is defined as system (14) which can drive the linear system (13) to $x(t)=0$ at $t=t_{F}$ in finite time. The state variables of system (14) converge to zero at $t=t_{F}$. The control law $u_{0}$ will maintain the required system states equilibrium after the terminal time, that is, $t>t_{F}$, provided that the forcing term $\delta(t)$ is removed at the terminal time; that is, $u_{0}=-K e(t)$ for $t>t_{F}$, where $K=B^{T} P$. Then, in order to reach the origin $e(t)=0$ and maintain at this point $t_{F}$ when $t>t_{F}$ in a finite time for system (13), [17] has designed an optimal control law as

$$
u_{0}= \begin{cases}-K e(t)+B^{T} \delta(t), & 0 \leq t \leq t_{F} \\ -K e(t), & t>t_{F}\end{cases}
$$

Theorem 8. For nonlinear system described by (5) whose dynamics satisfy Assumptions 1-3, the integral sliding mode surface is defined by taking (6). If the controller is designed as follows:

$$
u=u_{0}-M(t) \cdot \operatorname{sign}(s)
$$

where $M(t)>\left\|B^{+}\right\|(\alpha+\psi)-\eta$ with $u_{0}$ defined by (16), $\eta>0$ and $M(t)$ is a control gain. In the following, the proposed adaptive control method can ensure the establishment error state trajectory converges to the sliding manifold (6) in finite time.

Proof. Construct Lyapunov function

$$
V=\frac{1}{2} s^{T} s
$$

From (6) and Assumptions 1-3 by taking the time derivate of the integral sliding mode surfaces, we can get

$$
\begin{aligned}
\dot{V} & =s^{T} \dot{s} \\
& =s^{T}\left[B^{+}(f(y)-f(x))+u+B^{+} \phi+K e\right] \\
& =s^{T} \cdot\left[B^{+}(f(y)-f(x))+B^{+} \phi-M \cdot \operatorname{sign}(s)\right] \\
& \leq s^{T} \cdot\left[\left\|B^{+}\right\|\|(f(y)-f(x))\|+\left\|B^{+}\right\|\|\phi\|-M\right] \\
& \leq\|s\| \cdot\left[\left\|B^{+}\right\|(\alpha+\psi)-M(t)\right] \\
& \leq-\eta\|s\| .
\end{aligned}
$$

Therefore, a sliding mode control with respect to the system trajectories converges to zero in finite time according to Theorem 8 .

\section{Simulation Results}

In this section, the presented control algorithm is demonstrated in MATLAB/Simulink. Obviously, Assumptions 1 and 2 are satisfied by the system dynamics; thus we can design a robust integral sliding mode controller based on finite time stabilization using Theorem 8 . Then the initial value of the error system states $e=(1,0,-0.5)$ is supposed. Figure 2 depicts the simulation results of the states of error system (5). 


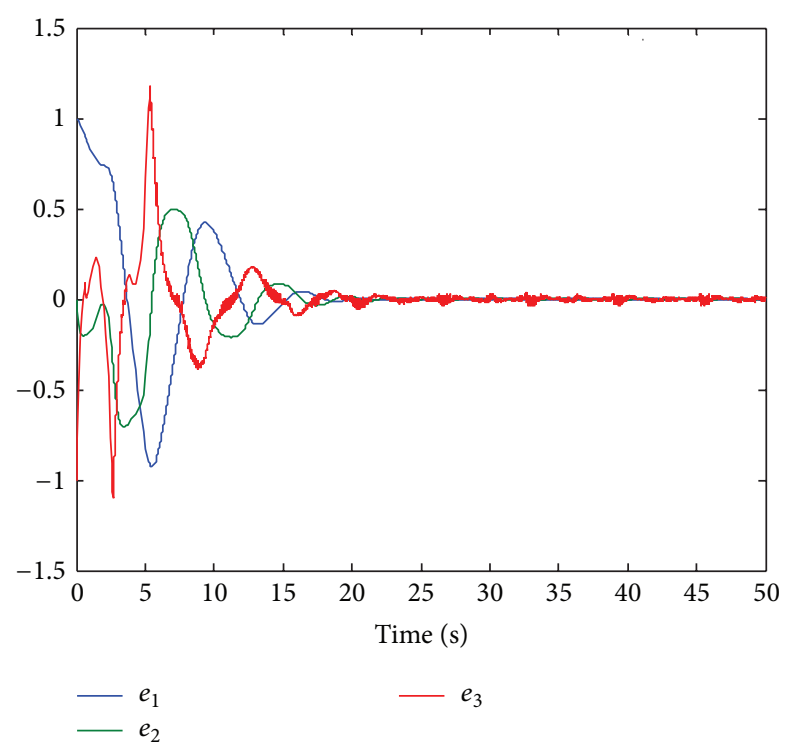

FIGURE 2: The error state response of the system (5).

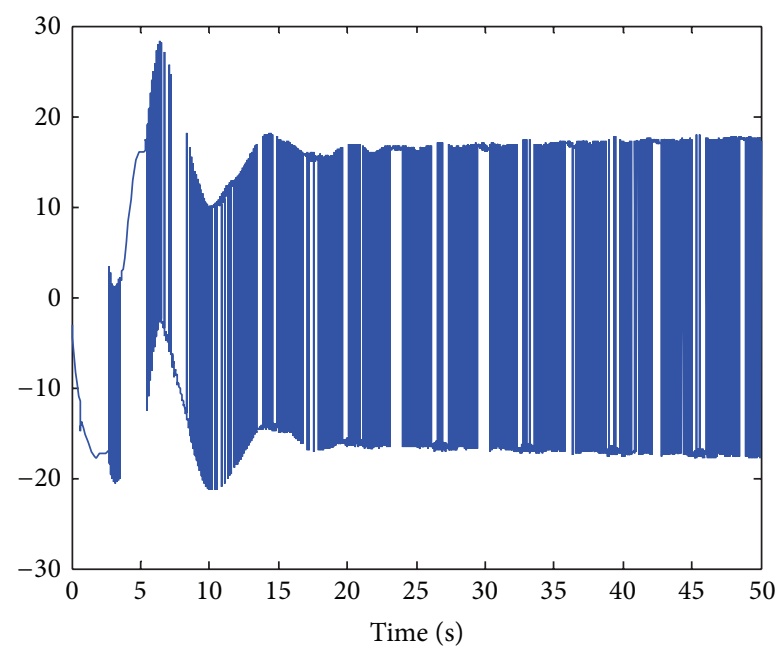

FIgURE 3: The response of control input for the closed-loop system (5).

From the figure, we can see that the synchronization errors $e_{1}, e_{2}$, and $e_{3}$ will converge to zero in the finite time. Figures 2 and 3 show the control input and the corresponding sliding surface $S(t)$. Suppose $\phi(t)=(0.1 \sin t, 0.5 \cos t,-0.3 \sin 2 t)^{T}$, $t_{F}=18$, and $Q=E_{3 \times 3}$. Consider $B=\left[\begin{array}{lll}0 & 0 & 1\end{array}\right]^{T}$. Moreover, $K=[-0.3,0.2,-0.16]$.

From Figure 2, we can see that the error system states responses rest on the ISMC surface although the unmatched uncertainties exist. It obviously ensures the synchronous error states for system (5) converge to zero in finite time from Figure 2. As shown in Figures 3 and 4, the control input response and the time response of ISMC surface are shown.

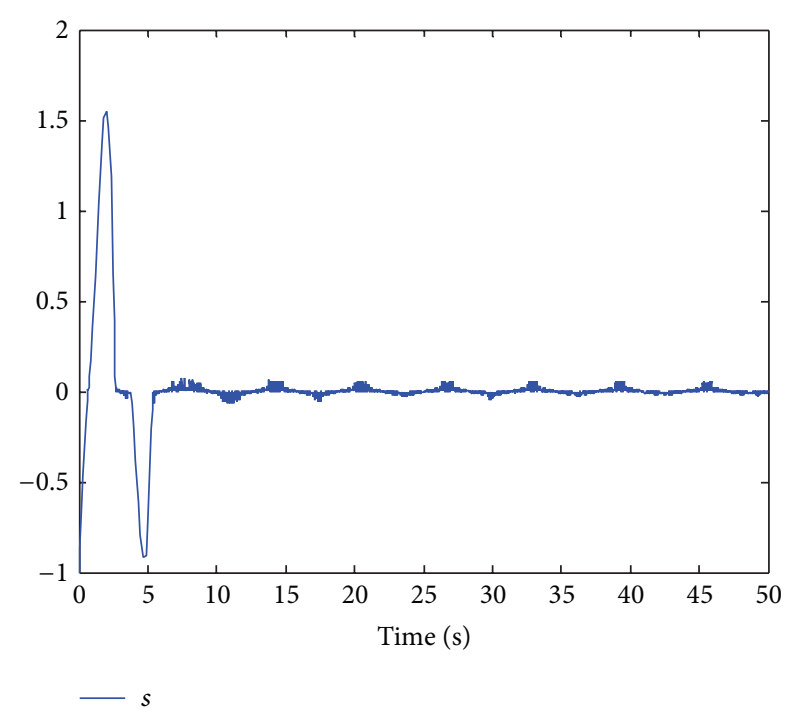

FIgURE 4: The integral sliding mode surface $s$.

\section{Conclusions}

A sliding mode control scheme based on integral sliding mode and optimal finite time convergent has been addressed in order to suppress the uncertain chaotic behavior in Lorenz system. The proposed method guarantees robustness by the Lyapunov stability method. The uncertainties of matching condition are not required to satisfy.

The method can be viewed as the finite time stabilization based on decomposition of matrix and integral sliding mode control. Finally, simulation results indicate that the proposed control techniques can achieve complete synchronization for Lorenz system in finite time. The further case of chaotic synchronization will be extended to discrete-time chaotic systems control approaches $[18,19]$ and it will bring big challenges for the chaotic synchronization.

\section{Conflict of Interests}

The authors declare that there is no conflict of interests regarding the publication of this paper.

\section{Acknowledgments}

This work is partially supported by the National Nature Science Foundation of China (no. 51378350). The authors also gratefully acknowledge the helpful comments and suggestions of the reviewers, which have improved the presentation.

\section{References}

[1] M. M. Arefi and M. R. Jahed-Motlagh, "Adaptive robust synchronization of Rossler systems in the presence of unknown matched time-varying parameters," Communications in Nonlinear Science and Numerical Simulation, vol. 15, no. 12, pp. 41494157, 2010. 
[2] Z.-M. Ge and C.-H. Yang, "Chaos synchronization and chaotization of complex chaotic systems in series form by optimal control," Chaos, Solitons and Fractals, vol. 42, no. 2, pp. 9941002, 2009.

[3] M. P. Aghababa, S. Khanmohammadi, and G. Alizadeh, "Finitetime synchronization of two different chaotic systems with unknown parameters via sliding mode technique," Applied Mathematical Modelling, vol. 35, no. 6, pp. 3080-3091, 2011.

[4] H. R. Karimi and H. Gao, "New delay-dependent exponential $H_{\infty}$ synchronization for uncertain neural networks with mixed time delays," IEEE Transactions on Systems, Man, and Cybernetics Part B: Cybernetics, vol. 40, no. 1, pp. 173-185, 2010.

[5] H. R. Karimi, M. Zapateiro, and N. Luo, "Adaptive synchronization of master-slave systems with mixed neutral and discrete time-delays and nonlinear perturbations," Asian Journal of Control, vol. 14, no. 1, pp. 251-257, 2012.

[6] H. R. Karimi, "A sliding mode approach to $H_{\infty}$ infinity synchronization of master-slave time-delays systems with Markovian jumping parameters and nonlinear uncertainties," Journal of the Franklin Institute, vol. 349, no. 4, pp. 1480-1496, 2012.

[7] H. R. Karimi and P. Maass, "Delay-range-dependent exponential $H_{\infty}$ synchronization of a class of delayed neural networks," Chaos, Solitons and Fractals, vol. 41, no. 3, pp. 1125-1135, 2009.

[8] Z. Wang, D. Ding, H. Dong, and H. Shu, " $H_{\infty}$ consensus control for multi-agent systems with missing measurements: the finitehorizon case," Systems and Control Letters, vol. 62, no. 10, pp. 827-836, 2013.

[9] Z. Wang, B. Shen, H. Shu, and G. Wei, "Quantized $H_{\infty}$ control for nonlinear stochastic time-delay systems with missing measurements," IEEE Transactions on Automatic Control, vol. 57, no. 6, pp. 1431-1444, 2012.

[10] S. Yin, S. X. Ding, X. Xie, and H. Luo, "A review on basic datadriven approaches for industrial process monitoring," IEEE Transactions on Industrial Electronics, vol. 61, no. 11, pp. 64186428, 2014.

[11] S. Yin, S. X. Ding, A. Sari, A. Haghani, and H. Hao, "Data-driven monitoring for stochastic systems and its application on batch process," International Journal of Systems Science, vol. 44, no. 7, pp. 1366-1376, 2013.

[12] S. Yin, H. Gao, and O. Kaynak, "Data-driven control and process monitoring for industrial applications-part I," IEEE Transactions on Industrial Electronics, vol. 61, no. 11, pp. 63566359, 2014.

[13] L. Wu, W. X. Zheng, and H. Gao, "Dissipativity-based sliding mode control of switched stochastic systems," IEEE Transactions on Automatic Control, vol. 58, no. 3, pp. 785-791, 2013.

[14] H. H. Choi, "A new method for variable structure control system design: a linear matrix inequality approach," Automatica, vol. 33, no. 11, pp. 2089-2092, 1997.

[15] X. Zhang, H. Su, L. Xiao, and J. Chu, "Robust sliding mode control based on integral sliding surfaces," in Proceedings of the American Control Conference (ACC '05), pp. 4074-4077, Portland, Ore, USA, June 2005.

[16] F. Castanos and L. Fridman, "Analysis and design of integral sliding manifolds for systems with unmatched perturbations," IEEE Transactions on Automatic Control, vol. 51, no. 5, pp. 853858, 2006.

[17] Z. V. Rekasius, "An alternate approach to the fixed terminal point regulator problem," IEEE Transactions on Automatic Control, vol. 9, pp. 290-292, 1964.
[18] Z. Wang, Y. Liu, G. Wei, and X. Liu, "A note on control of a class of discrete-time stochastic systems with distributed delays and nonlinear disturbances," Automatica, vol. 46, no. 3, pp. 543-548, 2010.

[19] Z. Wang, G. Wei, and G. Feng, "Reliable $H_{\infty}$ control for discrete-time piecewise linear systems with infinite distributed delays," Automatica, vol. 45, no. 12, pp. 2991-2994, 2009. 


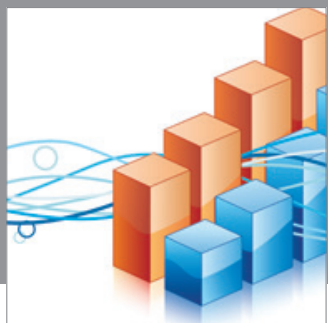

Advances in

Operations Research

mansans

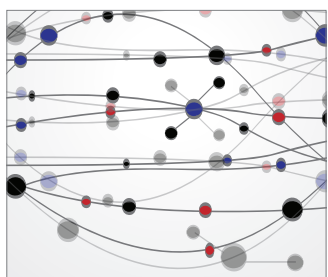

The Scientific World Journal
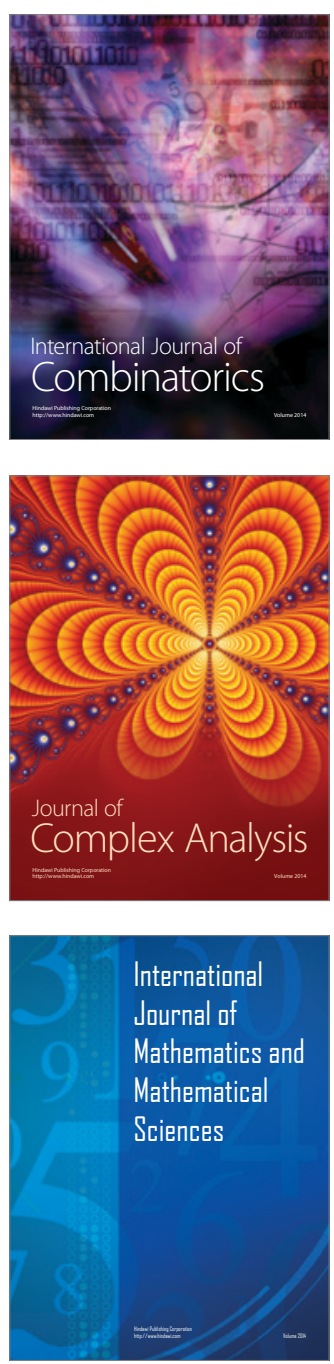
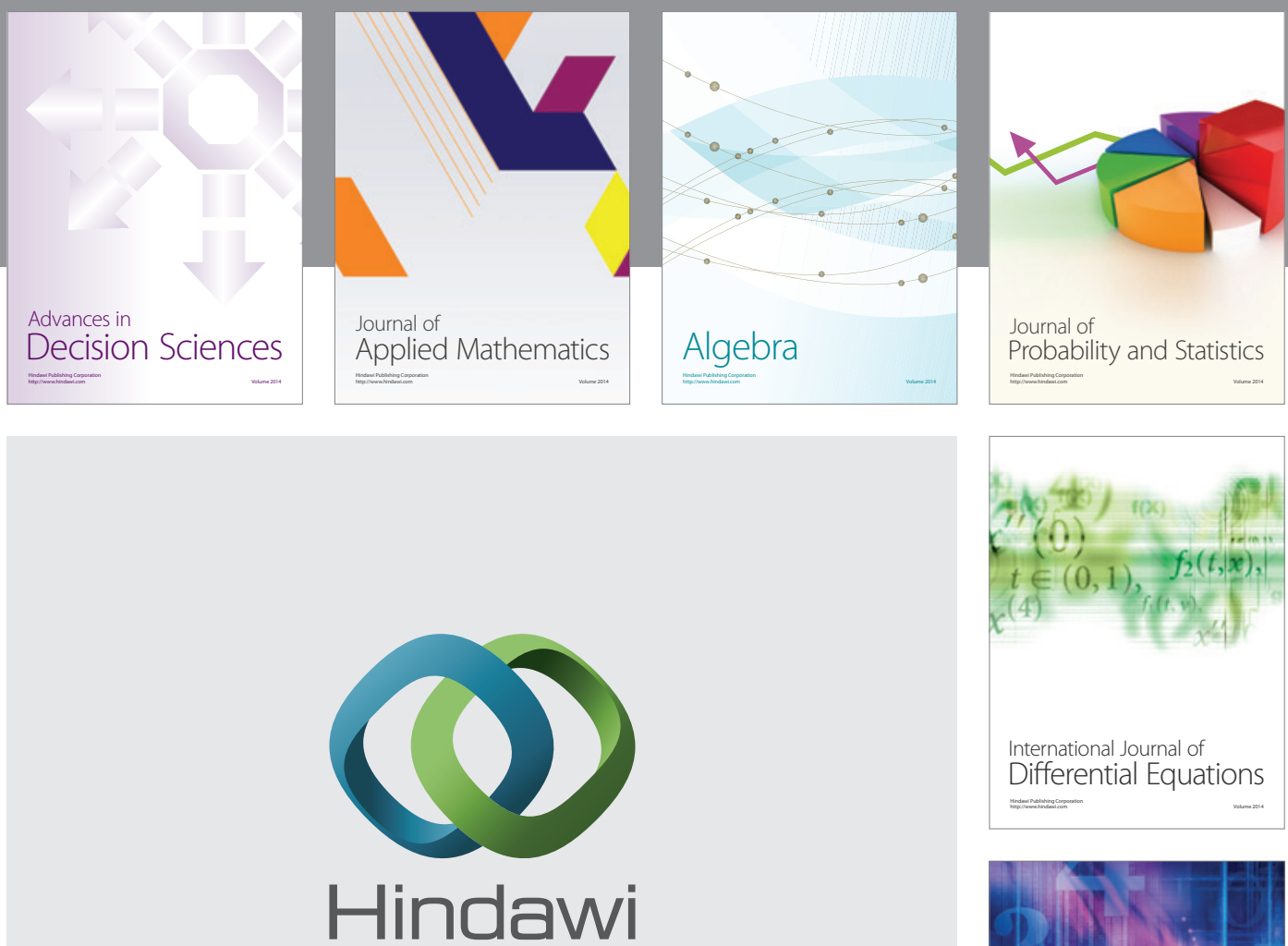

Submit your manuscripts at http://www.hindawi.com
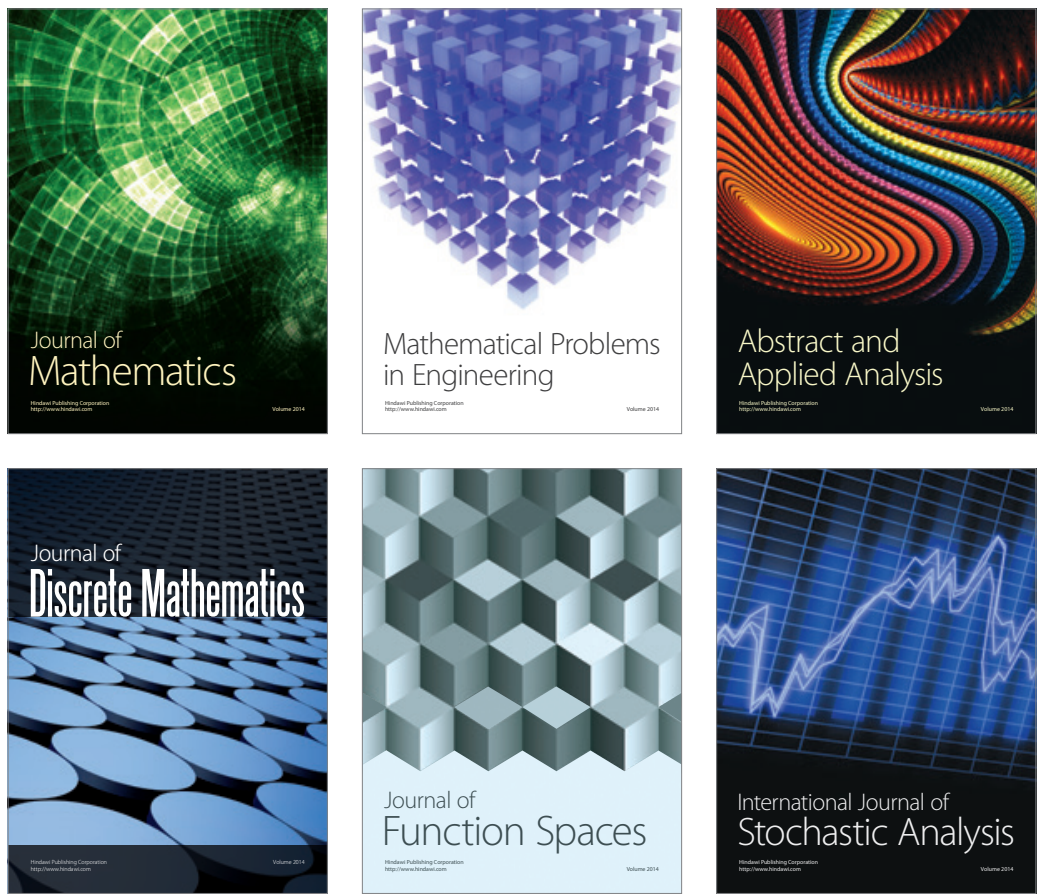

Journal of

Function Spaces

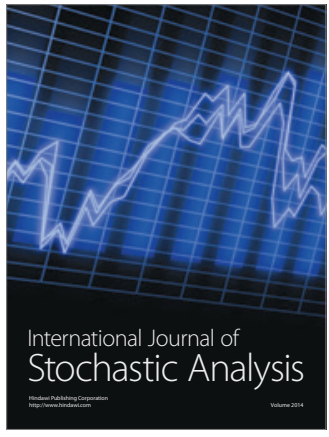

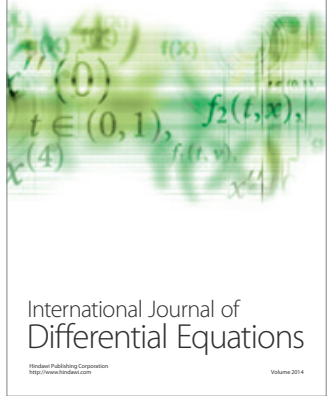
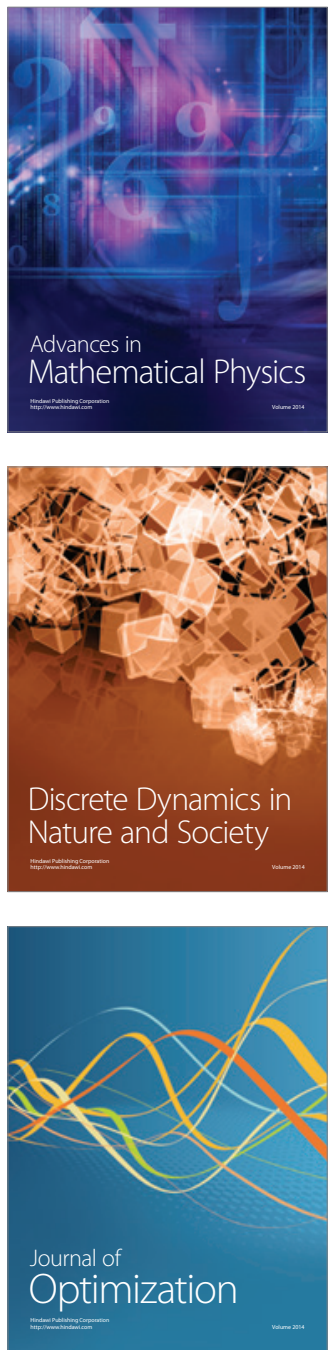